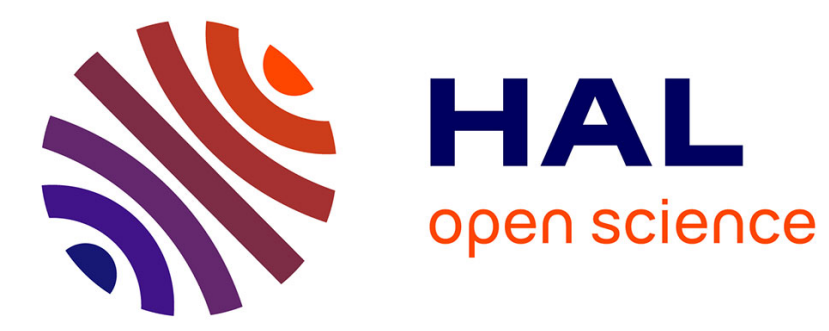

\title{
Modalités de la compensation des impacts sur la biodiversité en forêt
}

\author{
Marc Barré, Philippe Thiévent
}

\section{To cite this version:}

Marc Barré, Philippe Thiévent. Modalités de la compensation des impacts sur la biodiversité en forêt.

Revue forestière française, 2012, 64 (3), pp.257-262. 10.4267/2042/48434 . hal-03446917

\section{HAL Id: hal-03446917 \\ https://hal.science/hal-03446917}

Submitted on 24 Nov 2021

HAL is a multi-disciplinary open access archive for the deposit and dissemination of scientific research documents, whether they are published or not. The documents may come from teaching and research institutions in France or abroad, or from public or private research centers.
L'archive ouverte pluridisciplinaire HAL, est destinée au dépôt et à la diffusion de documents scientifiques de niveau recherche, publiés ou non, émanant des établissements d'enseignement et de recherche français ou étrangers, des laboratoires publics ou privés. 


\title{
BIODIUERSITE
}

\section{MODALITÉS DE LA COMPENSATION DES IMPACTS SUR LA BIODIVERSITÉ EN FORÊT}

\author{
Marc Barré - Philippe Thiévent
}

En France métropolitaine, la surface de terres agricoles ou d'espaces naturels artificialisée équivaut à un département tous les sept ans (soit environ $850 \mathrm{~km}^{2} / \mathrm{an}$ ) du fait de l'aménagement du territoire : voies de communication, extensions urbaines, zones d'activité, etc. (source IFEN).

Ceci se traduit par des impacts importants sur la biodiversité, par destruction des espèces, et par destruction ou dégradation de leur milieu de vie (pollution, fragmentation de l'espace, etc.).

Longtemps oubliée, la compensation des impacts de projets d'aménagement sur la biodiversité est revenue sur le devant de la scène : Grenelle de l'environnement, apparition de l'opérateur spécialisé “CDC Biodiversité”, groupe de travail "Éviter - Réduire - Compenser » animé par le ministère en charge de l'Écologie, positionnement de divers acteurs dont la Fédération des conservatoires d'espaces naturels.

Les gestionnaires d'espaces forestiers sont de plus en plus souvent confrontés à des demandes de mise en œuvre de mesures en faveur de la biodiversité sur les terrains qu'ils gèrent et exploitent, qui peuvent conduire à des inflexions des objectifs de gestion forestière, en particulier l'objectif de production.

\section{CONTEXTE RÉGLEMENTAIRE DE LA COMPENSATION DES IMPACTS SUR LA BIODIVERSITÉ}

La compensation des impacts sur la biodiversité est basée sur plusieurs textes réglementaires.

Pour l'autorisation de certains aménagements, la loi de 1976 (article L122-3 du Code de l'environnement) prévoit la réalisation d'une étude d'impact qui doit entre autres contenir "les mesures envisagées pour supprimer, réduire et, si possible, compenser les conséquences dommageables pour l'environnement et la santé ".

Bien que la loi de 1976 concerne l'environnement et, en son sein, la biodiversité en général, le statut réglementaire des espèces de faune et de flore est devenu un critère très important de l'évaluation des obligations de compensation. En effet, en cas de destruction d'espèces protégées ou, pour certaines, de leur habitat, l'autorisation du projet est soumise à l'avis consultatif du Conseil national de protection de la nature (CNPN). L'article L411-2 du Code de l'environnement alinéa 4 prévoit la possibilité de dérogations à l'interdiction de destruction des espèces et de leurs habitats " à condition qu'il n'existe pas d'autre solution satisfaisante et que la dérogation ne nuise pas au maintien, dans un état de conservation favorable, des populations des espèces concernées dans leur aire de répartition naturelle ", ce qui peut conduire à la mise en œuvre de mesures compensatoires.

Enfin, concernant le réseau de sites Natura 2000, l'article 6.4 de la directive 92/43/CEE dite «Directive Habitats » indique que "Si, en dépit de conclusions négatives de l'évaluation des 
MARC BarRé - PhiLIPPE ThiÉVent

incidences sur le site et en l'absence de solutions alternatives, un plan ou projet doit néanmoins être réalisé pour des raisons impératives d'intérêt public majeur, y compris de nature sociale ou économique, l'État membre prend toute mesure compensatoire nécessaire pour assurer que la cohérence globale de Natura 2000 est protégée».

Par ailleurs, le Code forestier fait mention de compensation pour défrichement via les boisements compensateurs en précisant une fourchette de ratios, mais n'impose pas de contrainte à la nature des boisements compensateurs en regard des enjeux écologiques de la forêt défrichée.

\section{QU'EST-CE QU'UNE MESURE COMPENSATOIRE ?}

\section{La nature des mesures compensatoires}

Tout d'abord, une mesure compensatoire doit être précédée d'actions d'évitement et de réduction des impacts avant d'envisager la compensation des impacts dits résiduels.

Les mesures compensatoires sont des actions qui conduisent à la création d'un gain de biodiversité. Elles sont définies de façon que ce gain de biodiversité corresponde au moins aux impacts résiduels du projet d'aménagement concerné, et dimensionnées de façon à les compenser.

Le résultat de ces mesures doit être une restauration ou une amélioration de l'état de conservation local ou régional des habitats et des espèces impactées, à la hauteur des atteintes.

Sur le terrain, il s'agit le plus souvent de mesures de restauration ou de réhabilitation d'habitats naturels ou d'habitats d'espèces. Dans ce cas, leur mise en œuvre passe par l'acquisition des terrains concernés ou par un conventionnement à l'amiable avec le propriétaire et ses ayants droit.

Mais il peut aussi s'agir de mesures jouant sur les effectifs locaux ou le succès de colonisation ou de reproduction des espèces : création de noyaux de population à partir d'individus sauvages ou produits ex situ, renforcement de populations, contrôle des prédateurs, création de corridors de déplacement. La compensation implique souvent un suivi et une gestion des espaces concernés sur des pas de temps longs (plusieurs dizaines d'années).

\section{La quantification des besoins de compensation}

Il n'existe pas actuellement de méthode formalisée au niveau national pour quantifier les impacts et les besoins de mesures compensatoires en France. Le ministère de l'Écologie a mis en place et anime un groupe de travail «Éviter - Réduire-Compenser » dont l'objet est d'élaborer une doctrine.

Néanmoins, il est couramment admis que plusieurs paramètres contribuent à augmenter les besoins de compensation face à un impact donné :

- le caractère aléatoire de la réussite des travaux de restauration, du fait de la complexité des milieux naturels, du manque de connaissances scientifiques et du manque de retour d'expérience en matière d'ingénierie écologique ;

- la durée de restauration. Lorsque l'impact de l'aménagement précède le moment où les mesures compensatoires ont atteint leur objectif écologique, les habitats et espèces passent par une phase pendant laquelle l'impact n'est pas encore compensé. C'est par exemple le cas en milieu forestier, du fait du temps long de la forêt ;

- la nature de l'action : pour compenser la destruction totale d'un habitat naturel, les surfaces de compensation seront différentes s'il s'agit de travaux lourds de réhabilitation à partir d'une situation dégradée, ou s'il s'agit de travaux légers d'amélioration d'une situation déjà proche du bon état recherché. 


\section{La nécessaire additionnalité des mesures compensatoires}

Pour que le gain apporté par les mesures compensatoires soit réel, il est indispensable que ces mesures ne se substituent pas aux actions menées dans d'autres cadres pour les mêmes habitats et espèces : actions de l'État dans le cadre du réseau Natura 2000 ou des plans nationaux de restauration d'espèces menacées, actions d'associations comme les conservatoires des espaces naturels, politiques environnementales des collectivités, politique européenne de "verdissement " de la politique agricole commune, etc. Par exemple, dans les forêts domaniales, l'État et son gestionnaire forestier, l'ONF, ont pris des engagements en matière de gestion forestière respectueuse d'enjeux de biodiversité (arbres morts ou sénescents, etc.). Les mesures compensatoires doivent pouvoir démontrer qu'elles s'ajoutent à ces efforts consentis par l'État, ce qui demanderait un suivi détaillé des mesures mises en œuvre tant par l'ONF que dans le cadre de la compensation.

\section{LES MESURES COMPENSATOIRES EN FORÊT}

\section{Quels habitats, quelles espèces ?}

Les mesures compensatoires en forêt concernent les habitats forestiers et les espèces qui utilisent des habitats forestiers pour tout ou partie de leur cycle biologique. II s'agira par exemple de :

- maintenir, restaurer ou recréer les habitats forestiers d'intérêt communautaire (ex : hêtraies à Aspérule odorante, forêts alluviales de bois durs) ;

- maintenir, restaurer ou recréer les habitats d'espèces protégées impactées (ex : réseau d'arbres sénescents pour les insectes saproxylophages, réseau de cavités d'arbres pour les chauves-souris forestières (voir encadré p. 260), éclaircies en forêt d'altitude pour favoriser la Myrtille au bénéfice du Grand Tétras) ;

- éventuellement, procéder à des créations ou renforcements de populations à partir d'individus collectés in natura ou multipliés ex situ.

Mais ces mesures compensatoires concernent aussi des espèces non forestières qui utilisent des milieux ouverts ou aquatiques actuellement insérés dans l'espace forestier. II pourra alors s'agir d'en augmenter l'étendue au détriment de la surface forestière (voir encadré sur le Fadet des laîches, p. 261).

\section{Les modalités de mise en œuvre des mesures compensatoires}

Le conventionnement entre l'opérateur des mesures compensatoires et le propriétaire forestier aura pour but de :

- garantir la pérennité de la vocation naturelle et forestière des sites ;

- modifier les pratiques de gestion et d'exploitation forestière pour les rendre compatibles avec les exigences écologiques des habitats et espèces ciblés ;

- autoriser l'opérateur à réaliser des aménagements écologiques (mares, nichoirs, etc.) et une gestion conservatoire des habitats.

\section{Les conséquences pour le gestionnaire forestier}

Suivant les exigences des habitats et des espèces ciblés, les prescriptions peuvent concerner tous les aspects de la gestion et de l'exploitation des massifs forestiers. Elles peuvent conduire à une révision du document d'aménagement. 
Mesures compensatoires en faveur des chauves-souris forestières : lecture des arrêtés préfectoraux pour les autoroutes $\mathrm{A}_{55}$ ( $\mathrm{Pau}$ - Langon) et $\mathrm{A}_{304}$ (Charleville-Mézière - Rocroi).

"Sécurisation, restauration et gestion conservatoire selon les exigences biologiques des espèces d'aires de repos et/ou sites de reproduction (formations alluviales et boisements caducifoliés) potentiels de chiroptères protégés sur une surface de 525 ha " (arrêtés interpréfectoraux $n^{\circ} 35 / 2008$ du 7 juillet 2008 et $n^{\circ}$ 65/2008 du 15 octobre 2008, portant autorisation de destruction d'espèces et d'habitats d'espèces animales protégées et de destruction d'espèces végétales protégées dans le cadre de la construction de l'autoroute A65 en Aquitaine).

"Un réseau d'îlots de sénescence et de vieillissement de 70 ha sera mis en place. Ce réseau, dont la délimitation précise devra être connue, sera essentiellement localisé en forêt domaniale. Il sera constitué d'îlots principaux de trois à cinq hectares chacun, connectés si possibles par des îlots secondaires de un à deux hectares. L'ensemble de ces îlots fera l'objet d'un suivi et d'une protection effective pendant trente ans à compter de leur désignation. " (Arrêté 2010/654 du préfet des Ardennes autorisant la capture ou l'enlèvement de spécimens d'espèces animales protégées, l'altération ou la dégradation de sites de reproduction ou d'aires de repos d'animaux d'espèces animales protégées dans le cadre du projet autoroutier A304).

À la lecture de ces deux arrêtés, on constate que les mesures compensatoires demandées pour les chauves-souris forestières concernent des surfaces considérables, mais sont formulées de façon très différente.

L'arrêté préfectoral pour l'A304 est particulièrement précis. Il impose un positionnement d'îlots de vieillissement et de sénescence dans l'espace forestier. On peut remarquer que la durée de trente ans est sans relation avec la durée de l'impact du projet autoroutier sur les chauvessouris, et qu'un vieillissement d'un peuplement de chênes du Nord de la France pendant trente ans ne conduit vraisemblablement pas à sa sénescence. On peut également être surpris de la demande d'une localisation essentiellement en forêt domaniale : faut-il y lire un espoir que le gestionnaire des forêts domaniales maintiendra ces îlots au-delà de l'obligation de 30 ans du maître d'ouvrage, ou bien qu'il sera plus facile de réussir le positionnement respectif des îlots dans l'espace au sein d'un vaste massif forestier propriété de l'État ?

L'arrêté interpréfectoral pour l'A65 est en revanche peu précis, sauf pour préciser que les peuplements visés sont feuillus dans un secteur dominé par le Pin maritime. Cette précision suffit-elle dans une région où les peuplements caducifoliés sont très peu exploités ?

\section{- Modification des peuplements}

L'objectif étant souvent de se rapprocher d'un habitat forestier " naturel », le sylviculteur pourra se voir prescrire des essences autochtones adaptées à la station forestière et, en plaine, des essences strictement feuillues. Ce sont aussi celles dont la présence est la plus compatible avec une végétation de sous-bois et une faune caractéristiques de l'habitat naturel recherché. Il s'agira aussi de diversifier les peuplements en intégrant un plus grand nombre et une plus grande proportion d'essences secondaires.

Le forestier pourra aussi se voir prescrire l'obligation d'utiliser des plants forestiers de souche et d'origine locales, dans le but d'éviter une pollution génétique. Pour les essences forestières les 
Mesures compensatoires en faveur du Fadet des laîches : lecture des arrêtés préfectoraux pour l'autoroute $\mathrm{A} 65$ (Pau - Langon) et la ligne à grande vitesse Sud-Europe-Atlantique (Tours - Angoulême - Bordeaux).

Le Fadet des laîches (Coenonympha oedippus) est un papillon diurne protégé et d'intérêt européen en voie de forte raréfaction en Europe. C'est un papillon des landes humides, dont la chenille se développe sur les touradons de Molinie. La population du massif landais est probablement en Europe celle dont l'état de conservation est le moins dégradé. Mais ses habitats de prédilection y ont été en grande majorité drainés et constituent les meilleures terres pour la production de Pin maritime.

"Sécurisation, restauration et gestion conservatoire selon les exigences biologiques des espèces, [...] d'habitats de repos et/ou de reproduction de Fadet des laîches sur une surface de 216,1 ha " (arrêtés interpréfectoraux n 35/2008 du 7 juillet 2008 et $n^{\circ}$ 65/2008 du 15 octobre 2008, portant autorisation de destruction d'espèces et d'habitats d'espèces

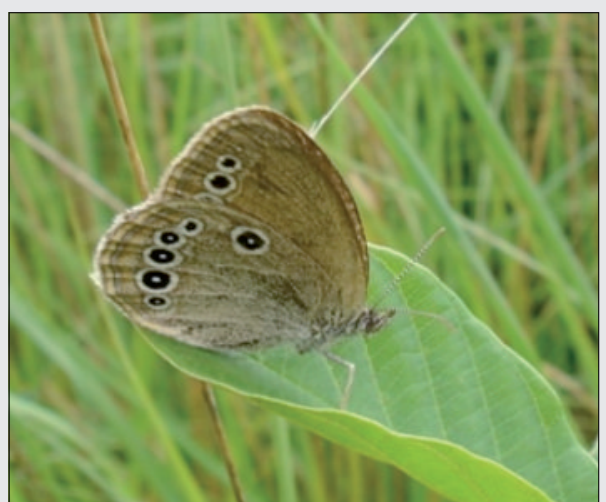

Photo 1 : Fadet des Laîches Photo Pierre Boyer - CDC Biodiversité animales protégées et de destruction d'espèces végétales protégées dans le cadre de la construction de l'autoroute A65 en Aquitaine).

"Acquisition de 100 hectares dans le massif forestier de la Double Saintongeaise, les parcelles forestières non replantées suite aux différentes tempêtes seront visées en priorité " (arrêté interpréfectoral 01/2010 du 5 juillet 2010 portant autorisation de destruction d'espèces et d'habitats d'espèces animales protégées et de destruction d'espèces végétales protégées dans le cadre des travaux de défrichement et d'archéologie préventive liés à la construction de la ligne à grande vitesse Sud-Europe-Atlantique).

On constate que les surfaces à restaurer pour le Fadet des laîches sont importantes dans les deux cas. Si l'arrêté interpréfectoral pour l'A65 est peu précis quant à la nature des terrains éligibles et des mesures de restauration, il est clair dans celui de la LGV SEA qu'il s'agit de parcelles ayant un statut forestier et qu'il s'agit de défricher. Toute la question est de savoir si des boisements compensateurs seront nécessaires, et quel sera leur impact sur l'habitat du Fadet.

plus communes de notre pays (colonisation récente du territoire après la dernière glaciation, espèces anémochores à répartition continue), et dans un contexte de réchauffement climatique (les adaptations éventuelles aux conditions locales sont-elles remises en cause, ces conditions étant amenées à changer très fortement ?), cette disposition peut être discutée.

\section{- Conduite et exploitation des peuplements : vers un jardinage?}

La coupe à blanc d'un peuplement sur des surfaces importantes conduisant à une modification brutale des conditions d'ensoleillement, de température et d'humidité, il est fréquemment demandé au forestier d'y renoncer en faveur de parcelles de petite dimension, voire d'une gestion arbre par arbre qui concilie exploitation et maintien des conditions forestières, ou de 
favoriser la présence d'un sous-étage diversifié. Poussée à l'extrême, cette logique aboutit à un jardinage fin du peuplement qui demande une refonte du système de gestion de la forêt, mais aussi des modes d'exploitation et de valorisation des bois.

Enfin, des contraintes peuvent être apportées aux modalités d'abattage et de débardage des bois, de façon à éviter la dégradation des habitats et le dérangement ou la destruction des espècescibles : dates d'intervention compatibles avec les périodes de sensibilité au dérangement, suppression des engins lourds en zone humide ou sur les berges des cours d'eau, conditions à la création de pistes de débardage, etc. Certaines prescriptions peuvent concerner les pratiques de chasse.

\section{- Surfaces exclues de l'exploitation forestière}

Les exigences écologiques des habitats et espèces peuvent conduire à exclure certaines parties du massif forestier de toute exploitation des peuplements, voire d'autres modes de valorisation comme la chasse. Les mesures les plus notables de ce point de vue sont la création d'îlots de sénescence pour les chauves-souris (voir encadré sur les mesures en faveur des chauves-souris forestières, p. 260) et du cortège saproxylophage, ou la création de clairières.

Il faut aussi citer les espèces dont la raréfaction est en partie due au boisement ou au drainage, plus ou moins ancien, de leur habitat. La restauration de leur habitat passe par un défrichement, avec demande d'autorisation et, éventuellement, ce qui peut sembler paradoxal, l'obligation de réaliser des boisements compensateurs (voir encadré sur le Fadet des laîches, p. 261).

Marc BARRÉ - Philippe THIÉVENT CDC Biodiversité 102 rue Réaumur F-75002 PARIS

(m.barre.cdcbiodiv@forestiere-cdc.fr)

\section{mODALITES DE LA COMPENSATION DES ImPACTS SUR LA BIODIUERSITE EN FORÊT [RÉsumé]}

La compensation des impacts sur la biodiversité est basée sur la loi de 1976 portant sur les études d'impacts (Code de l'environnement L122-4), sur la directive Habitats (article 6.4) et sur la réglementation de protection d'espèces de faune et de flore. Les mesures compensatoires sont des actions positives pour la biodiversité et à caractère additionnel. Il s'agit sur le terrain de la restauration d'habitats forestiers et d'habitats d'espèces, mais aussi d'opérations de renforcement des populations des espèces ciblées. Les mesures doivent s'additionner aux engagements déjà pris par le gestionnaire sur les mêmes espaces. Leur mise en œuvre passe par la sécurisation foncière à l'amiable des parcelles forestières concernées par acquisition ou conventionnement avec leur propriétaire. En forêt, elle a des répercussions diverses sur la gestion et l'exploitation forestière, parmi lesquelles une valorisation en dehors des itinéraires sylvicoles classiques, voire dans certains cas une non valorisation (îlots de sénescence, extension des milieux ouverts au sein du massif, etc.). Des exemples d'arrêtés préfectoraux prescrivant des mesures en faveur des chauves-souris forestières et du Fadet des laîches (Coenonympha oedippus) sont discutés.

\section{TERMS OF COMPENSATION FOR IMPACTS ON BIODIUERSITY IN FORESTS [Abstract]}

Compensation for impacts on biodiversity is based on the 1976 Law on impact surveys (Environmental Code Article L.122-4), the Habitats Directive (Article 6.4) and regulations pertaining to the protection of species flora and fauna. Compensatory measures are positive, supplementary actions in favour of biodiversity. They involve restoration in the field of forest and species habitats, as well as operations aimed at strengthening target species populations. Such measures must be additional to commitments already undertaken by the manager in relation to the same locations. Their implementation requires the tenure of the forest plots involved to be amicably secured either by acquisition or by entering into contracts with their owners. In forestry, these measures have various impacts on management and exploitation, including value added obtained from non-conventional silvicultural means, or even from outright non-exploitation (old growth islands, extension of clearings within the forest, etc.). Examples of Prefecture decrees prescribing measures in favour of forest bats and False Ringlet butterflies (Coenonympha oedippus) are discussed. 